\title{
Osteonecrosis as the presenting feature in a child with acute lymphoblastic leukaemia
}

\author{
Sern Chin Lim, Bushra Johari, Swee Ping Tang \\ Lim SC, Johari B, Tang SP. Osteonecrosis as the presenting feature in a child with acute lymphoblastic leukaemia. Malays Fam Physician. \\ 2021;16(3);115-118. https://doi.org/10.51866/cr1176
}

\section{Keywords:}

Osteonecrosis, acute

lymphoblastic leukaemia,

joint pain

\section{Authors:}

\section{Lim Sern Chin \\ (Corresponding author) MD (UKM), FRCPCH (UK) \\ Faculty of Medicine, Universiti Teknologi MARA (UiTM), Sungai Buloh, Selangor, Malaysia Email: sernchin@gmail.com}

\section{Bushra Johari}

MBBS (IIUM), Master of Radiology (UM), FRCR (UK)

Faculty of Medicine, Universiti Teknologi MARA (UiTM), Sungai Buloh, Selangor, Malaysia

\section{Tang Swee Ping}

MBBS (UM), FRCPCH (UK)

Department of Paediatrics, Selayang

Hospital, Ministry of Health, Batu

Caves, Selangor, Malaysia

\begin{abstract}
A seven-year-old girl presented with pain in multiple joints and constitutional symptoms over a period of four months. There were no significant clinical findings apart from joint tenderness. Blood test results did not indicate any specific pathology and initial radiology imaging was normal. Subsequent careful examination of her X-ray images led to an MRI of her left knee, which revealed acute osteonecrotic changes. A following whole-body MRI examination demonstrated multifocal bony lesions. Bone marrow examination conclusively diagnosed acute lymphoblastic leukaemia (ALL). Acute osteonecrosis has classically been described as a complication of treatment in children with ALL and has not been recognised as a presenting feature until recently.
\end{abstract}

\section{Case Report}

A seven-year-old girl presented with complaints of pain in multiple joints for the past four months. She first developed pain over her right shoulder following a trivial fall. She was seen at an urgent care center and treated with an arm sling for a presumed hairline fracture of her right humeral neck. The pain gradually resolved and a subsequent $\mathrm{X}$-ray two weeks later was reportedly normal. Later that week, however, she began complaining of pain in multiple joints, initially over the left shoulder and then over both knees. She also developed intermittent low-grade fever, and loss of appetite and weight. Her joint pains were intermittent, with no specific pattern.

A month later she presented to another hospital due to her complaints. Blood investigations showed a white cell count of $5.2 \times 10^{9} / \mathrm{L}$ (neutrophils $35.9 \%$, lymphocytes $54.6 \%$ ), haemoglobin of $13.2 \mathrm{~g} / \mathrm{dL}$, platelets of $479 \times 10^{9} / \mathrm{L}$ and an erythrocyte sedimentation rate (ESR) of $39 \mathrm{~mm} / \mathrm{hr}$. Multiple X-rays, including chest and left knee, were performed and reported as normal (Figure 1a). She was diagnosed with a viral infection and discharged after a brief hospital stay. The child continued to have intermittent knee pain with low grade fever, however, disrupting her sleep and daily activities. Her parents brought her to multiple paediatricians, where they were repeatedly reassured and the child was treated symptomatically with paracetamol. She did not receive any other medications, including NSAIDs or steroids.

At presentation to our centre, the child was afebrile but had severe left knee pain with inability to ambulate. There was no swelling or erythema over her left knee, despite her being in severe pain with limitation of movement. Further systemic examination was normal. A provisional diagnosis of inflammatory arthritis with possible malignancy was made. Repeated blood investigations showed a white cell count of $7.7 \times 10^{9} / \mathrm{L}$, haemoglobin of $11 \mathrm{~g} /$ $\mathrm{dL}$, platelets of $524 \times 10^{9} / \mathrm{L}$, ESR of $17 \mathrm{~mm} /$ $\mathrm{hr}$, C-reactive protein of $120 \mathrm{mg} / \mathrm{L}$, lactate dehydrogenase of $556 \mathrm{IU} / \mathrm{L}$, and negative antinuclear antibodies and rheumatoid factors. Peripheral blood films showed large, atypical lymphocytes with no blasts.

A repeat X-ray of the left knee showed a suspicious appearance (Figure 1b). Following that, magnetic resonance imaging (MRI) of metaphysis and epiphysis of the distal femur and proximal tibia, exhibiting features consistent with osteonecrosis (Figure 2). Based on the MRI findings, a differential of acute leukaemia or chronic recurrent multifocal osteomyelitis was made. After a multidisciplinary discussion, a whole-body MRI was performed, which showed multiple lesions in the axial and appendicular bones (Figure 3). Subsequent bone marrow analysis was diagnostic of acute lymphoblastic leukaemia. the left knee revealed multifocal lesions at the 


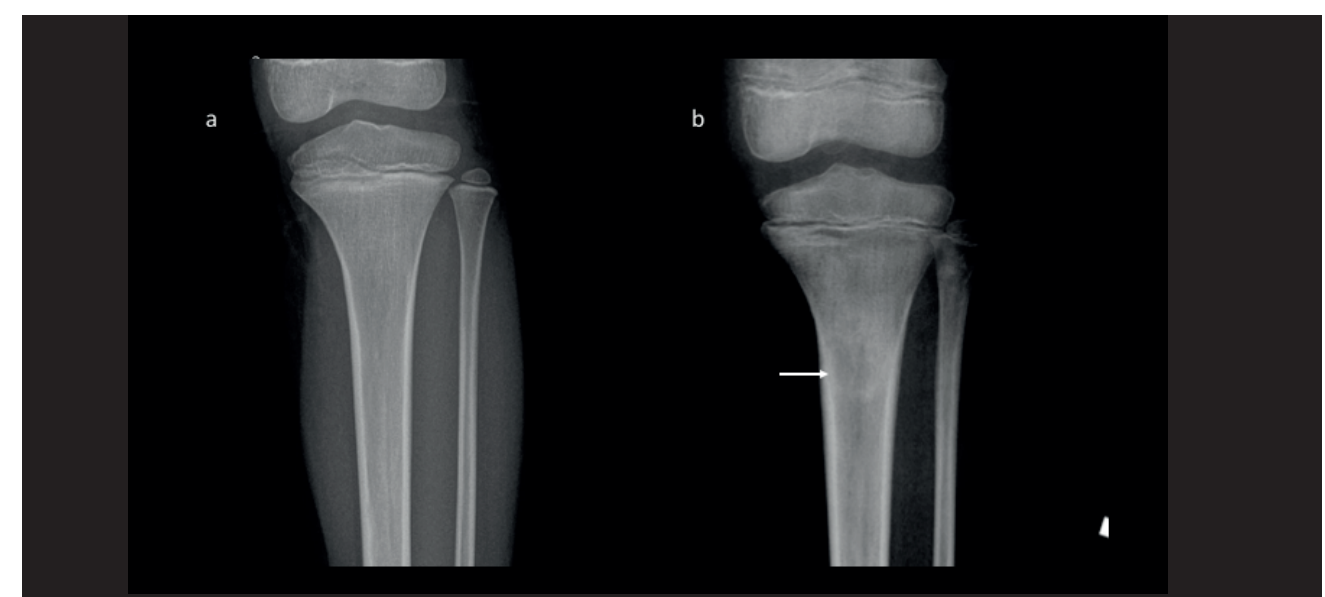

Figure 1. X-rays of seven-year-old girl with joint pain. (a) Initial X-ray (zoomed-in) of the left knee joint and the proximal tibia and fibula showed no significant findings. (b) A repeat X-ray three months later showed subtle lucency with surrounding sclerosis (arrow) at the proximal tibia.

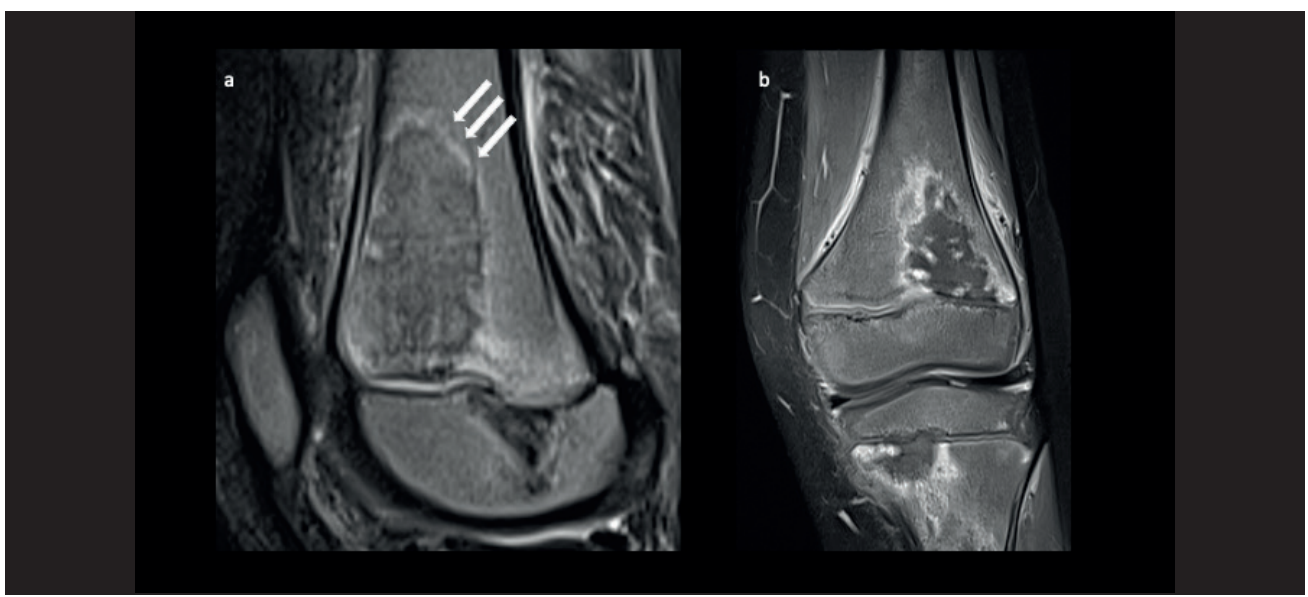

Figure 2. MRI of the left knee of a seven-year-old girl with joint pain. T2 TIRM (29/5500) in sagittal section (a) showing geographical lesions at distal femur, with some areas exhibiting serpiginous double-line sign (arrows) of inner high-signal-intensity area, the reactive interface or zone of creeping substitution, and the outer low-signal-intensity sclerotic rim, favouring osteonecrosis. T1FS contrast-enhanced (14/726) coronal image (b) showed marginal enhancement of the lesions in the distal femur and proximal tibia.

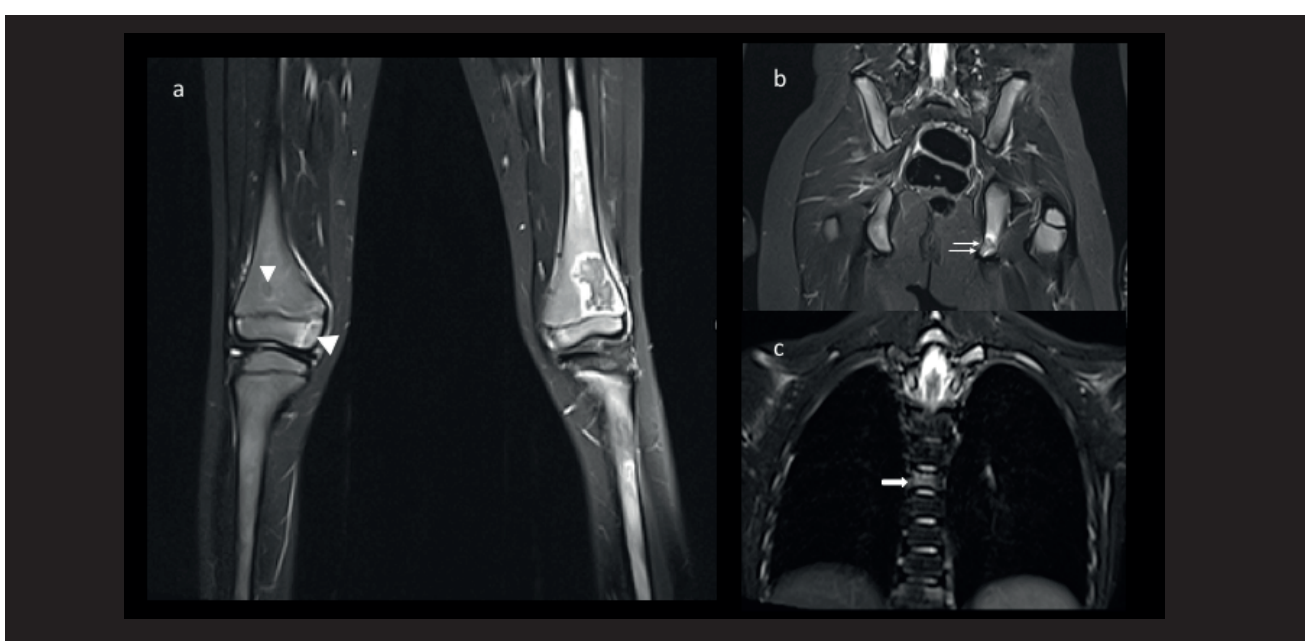

Figure 3. Selected T2 TIRM images from MRI whole-body scan of seven-year-old girl with joint pain. Apart from the lesions around the left knee, more lesions were observed in the rest of the axial and appendicular skeleton. Shown here are the lesions in the (a) right femur (arrowheads), (b) left ischium (double arrows) and (c) thoracic vertebra (arrow). 


\section{Discussion}

Acute lymphoblastic leukaemia (ALL) is the most common malignancy in childhood, accounting for approximately $30 \%$ of all childhood malignancies under the age 15 years and constituting nearly $80 \%$ of all childhood leukaemias. ${ }^{1}$. Acute leukaemia in children is known to present in myriad ways, ranging from non-specific constitutional symptoms to more concerning ones such as bleeding tendencies, generalised lymphadenopathy or hepatosplenomegaly.

Musculoskeletal manifestations in children with ALL are common and have been reported as the initial presentation in up to $30 \%$ of cases. $^{2}$ Symptoms range from joint pains or swelling to more persistent bone pain. Often, clinical findings are non-specific and may be difficult to differentiate from inflammatory arthritis. ${ }^{2,3}$ The classical diurnal pattern of pain (inflammatory pain worse in the mornings and nocturnal pain in malignancy or infection) may sometimes not be obvious in young children, as evident in our patient. The four-month history of constitutional symptoms and absence of objective clinical findings of arthritis were uncharacteristic for inflammatory arthritis or other benign musculoskeletal conditions. Features that typically raise suspicion of malignancies like lymphadenopathy and organomegaly may not be consistently present and, indeed, were notably absent in our patient. It is therefore imperative to consider possible malignancies in children who present with persistent musculoskeletal complaints, especially if the presentation is atypical.

The most important initial test to diagnose acute leukaemia is a full blood picture, which typically demonstrates pancytopenia with the presence of blasts. This blood film may, however, be entirely normal in the early phases of the disease. Thus, a normal blood count, even in the absence of lymphadenopathy or organomegaly, should not exclude leukaemia if the clinical picture is suspicious. ${ }^{2}$ In such situations, a bone marrow examination is warranted.

Musculoskeletal radiographic abnormalities at diagnosis are well recognised in paediatric ALL, being reported in approximately $80 \%$ of cases in some series. ${ }^{3,4}$ The most common are metaphyseal lucencies $(7.5-70 \%$, according to published data). ${ }^{3}$ Other reported abnormalities include periosteal reactions, osteolytic lesions, osteopenia and pathological fractures from marrow infiltration. ${ }^{3}$ Osteonecrosis per se is not a generally known presenting feature of ALL and has only been recognised in recent literature. $^{5}$

Osteonecrosis varies in radiographic presentation depending on the stage, with normal radiographs in the earliest stages, progressing to subtle non-specific lucencies and collapse of the bone in the later stages. MRI remains the imaging modality of choice in the evaluation of osteonecrosis. The doubleline sign of parallel hyper- and hypointense serpiginous lines representing reactive interfaces or zones of creeping substitution is considered diagnostic of osteonecrosis on MRI. ${ }^{6}$ Our case demonstrates the importance of not dismissing persistent musculoskeletal symptoms even when initial radiographs are normal.

Osteonecrosis in children has been classically described as a complication in the treatment of ALL. Incidence ranges from $1.8 \%$ to $8.9 \%$ in multiple studies ${ }^{4}$; this is likely an underestimate, as many studies examined only symptomatic children. Prospective MRI studies performed in all ALL children, regardless of symptoms, quote a higher incidence $15.5 \%$ to $24 \%$. $^{7}$ The risk is higher in children older than 10 years with exposure to corticosteroids. ${ }^{4,7}$ The exact aetiology of osteonecrosis in children with ALL remains uncertain and the presence of early osteonecrosis in patients like ours who have not been exposed to corticosteroids or chemotherapy suggests that the inherent nature of ALL itself may play a role in the formation of these lesions. ${ }^{8}$

Although MRI was very useful in our patient, clinicians should be mindful that MRI is not mandatory prior to a marrow examination. We would recommend a bone marrow examination in any child with a suspicion of a haematological malignancy, even without an MRI. Our case highlights the need for a thorough investigation in children with significant musculoskeletal symptoms despite scant clinical findings.

\section{Ethics approval}

This case report has been approved for publication by the Medical Research and Ethic Committee, National Institute of Health, Malaysia (NMRR-20-1414-55268)

\section{Funding}

This paper is investigator initiated and received no external funding. 


\section{Conflicts of Interest}

The authors declare that they have no conflicts of interest.

\section{Acknowledgement}

The authors would like to thank the Director General of Health Malaysia for the permission to publish this paper.

\section{How does this paper make a difference to general practice?}

- Musculoskeletal manifestations in children with ALL are well known and can mimic arthritis or even osteomyelitis.

- Any persistent musculoskeletal symptoms, especially with atypical features such as constitutional symptoms or disproportionate pain, warrant a thorough investigation even if clinical findings are sparse.

- Plain radiographs may initially be normal in osteonecrosis and the presence of persistent and significant bone pain is an indication for more discriminatory tests, such as a CT scan or MRI.

- Absence of blasts on peripheral blood films does not exclude leukaemia, as these films may be normal in the early phase of acute leukaemia.

\section{References}

1. Steliarova-Foucher E, Colombet M, Ries LAG, et al. International incidence of childhood cancer, 2001-10: a population-based registry study. The Lancet Oncology 2017; 18: 719-731.

2. Mitchell C, Hall G, Clarke RT. Acute leukaemia in children: diagnosis and management. BMJ; 338. Epub ahead of print 11 June 2009. DOI: 10.1136/bmj.b2285.

3. Rogalsky RJ, Black GB, Reed MH Orthopaedic manifestations of leukemia in children. J Bone Joint Surg Am 1986; 68: 494-501.
4. Kunstreich M, Kummer S, Laws H-J, et al. Osteonecrosis in children with acute lymphoblastic leukemia. Haematologica 2016; 101: 1295-1305.

5. Krull K, Kunstreich M, Bronsema A, et al. Osteonecrosis in children with acute lymphoblastic leukemia at initial diagnosis and prior to any chemotherapy. Leukemia \& Lymphoma 2019; 60: 78-84.

6. Murphey MD, Foreman KL, Klassen-Fischer MK, et al. From the radiologic pathology archives: Imaging of osteonecrosis: Radiologicpathologic correlation. Radiographics : a review publication of the Radiological Society of North America, Inc 2014; 34: 1003-1028.
7. Ribeiro RC, Fletcher BD, Kennedy W, et al. Magnetic resonance imaging detection of avascular necrosis of the bone in children receiving intensive prednisone therapy for acute lymphoblastic leukemia or non-Hodgkin lymphoma. Leukemia 2001; 15: 891-897.

8. Mostoufi-Moab S, Halton J. Bone morbidity in childhood leukemia: epidemiology, mechanisms, diagnosis, and treatment. Curr Osteoporos Rep 2014; 12: 300-312. 